\title{
Reliability-Based Design Optimization of Trusses with Linked-Discrete Design Variables using the Improved Firefly Algorithm
}

\author{
Nader M. Okasha \\ Civil Engineering Department \\ University of Hail \\ Hail, Saudi Arabia \\ n.okasha@uoh.edu.sa
}

\begin{abstract}
In this paper, an approach for conducting a Reliability-Based Design Optimization (RBDO) of truss structures with linked-discrete design variables is proposed. The sections of the truss members are selected from the AISC standard tables and thus the design variables that represent the properties of each section are linked. Latin hypercube sampling is used in the evaluation of the structural reliability. The improved firefly algorithm is used for the optimization solution process. It was found that in order to use the improved firefly algorithm for efficiently solving problems of reliability-based design optimization with linked-discrete design variables; it needs to be modified as proposed in this paper to accelerate its convergence.
\end{abstract}

\section{Keywords-Fierfly; RBDO; LHS; truss; linked-discrete}

\section{INTRODUCTION}

When design variables of an optimization problem can assume only some predetermined values, these variables are discrete. One of the approaches used to handle discrete design variables in engineering problems is to solve these problems as if the design variables are continuous and adjust the optimal design to the nearest discrete values [1]. The interest in optimization problems with discrete design variables in structural engineering applications dates back to the 1960s. Early reviews about the development in this topic can be found in [2]. More recent treatments of discrete variables in structural optimization of trusses can be found in the literature [3-6].

In the case of trusses, only one section property is typically treated in the literature as a design variable for each structural member, namely the cross sectional area. In these cases, the design variables are treated as independent of each other. However, the optimization of most structural systems requires using linked-discrete design variables. Practically, a structural designer selects the sections from the American Institute of Steel Construction (AISC) standard tables [7]. If a section is selected from these tables, the section properties must all take the values pertaining to that section. Accordingly, if each section property is treated as a design variable, they are naturally linked. Treating these design variables as independent will surely lead to unacceptable results. In [8] the approaches that had been used to solve problems of this class of optimization to that date were reviewed. In [9] strategies to reduce the large computing time required to solve optimization problems of steel structures with standard sections were suggested.

The reliability-based design optimization problem domain is complex and requires the application of robust search and optimization techniques to arrive at a near-global optimal solution [6]. In [10] an overview of various reliability-based optimization design approaches which were tested on a benchmark constituted of four examples using mathematical and finite element models, with different levels of difficulties, was given. One of the methods that have been efficiently used in solving problems of structural reliability in various engineering applications is the Latin hypercube sampling [6; 11-13]. The optimization of practical structural systems involves a problem with discrete-linked design variables, and is subjected to nonlinear implicit random constraints. Furthermore, the gradients cannot be explicitly expressed. Therefore, gradient-based optimization algorithms are unlikely to be suitable for solving this type of problems. Global search methods can overcome most of the difficulties that gradientbased algorithms cannot handle.

Many global search algorithms have been developed over the past few decades. Some of the most promising algorithms are nature-inspired, and they are called metaheuristic algorithms. Among these algorithms are Genetic Algorithms (GA) [14], which simulate the process of natural selection, and the Firefly Algorithm (FA) [15], which mimics the social behavior of fireflies based on their flashing characteristics. Also, Simulated Annealing (SA) [16] models the physical process of heating a material and then slowly lowering the temperature to decrease defects, thus minimizing the system energy. Even though SA and GA methods do not guarantee an optimum solution, they provide a reasonable solution at an acceptable computational cost [17]. In [18] a real coded genetic algorithm named MI-LXPM for solving integer and mixed integer constrained optimization problems was developed. The optimum design of trusses was investigated in the literature using GA [4,6], SA [9], and FA [3,5]. However, the reliabilitybased design optimization of trusses considering linkeddiscrete design variables using FA has not been attempted yet. 
In this paper, an approach for conducting a reliability-based design optimization of truss structures with linked-discrete design variables is proposed. The sections are selected from the AISC standard tables and thus the design variables that represent the properties of the section are linked and discrete. If a section is selected from the tables, the design variables must take the values pertaining to that section. The Improved Firefly Algorithm (IFA) is used for the optimization. Latin hypercube sampling is used to determine the structural reliability. It is found that despite the strength of the IFA in solving deterministic optimization problems, it is unstable when used in probabilistic and reliability-based optimization problems. A modification to the IFA is proposed in order to stabilize it for use in probabilistic and reliability-based optimization problems. The modification is tested on a planner truss and a space truss. The performance of the IFA is investigated with and without the proposed modification in both truss examples. Then, the approach for the reliability-based design optimization with discrete-linked design variables is tested on a truss bridge and the results are compared with those of GA and SA.

\section{NOMINAL STRENGTH OF TRUSS MEMBERS UNDER TENSILE LOADS}

Even though a ductile steel member without holes and subjected to tensile loads can resist loads larger than its yielding capacity, due to the effect of strain hardening, the reaching of strain hardening in a member results in unacceptable deformations. Therefore, sections of a tension member are deemed to resist loads up to their yielding capacity. The presence of bolt holes may cause the member to fail by fracture at the net section through the holes. This fracture load may be smaller than the yielding capacity of the gross section. Accordingly, the AISC [7] states that the nominal strength of a tension member, $P_{n t}$, may be the smaller of the tensile yielding strength of the member, $P_{n y}$, and the tensile rupture strength of the member, $P_{n}$, given by (1) and (2), respectively [7]:

$$
\begin{gathered}
P_{n y}=F_{y} A_{g} \\
P_{n r}=F_{u} A_{e}
\end{gathered}
$$

In the preceding expressions, $F_{y}$ and $F_{u}$ are the specified minimum yield and ultimate tensile stresses, respectively, $A_{g}$ is the gross area of the cross section of the member, and $A_{e}$ is the effective net area that is assumed to resist the loads at the section through the holes. The effective net area is related to the net area, $A_{n}$, by [19]:

$$
A_{e}=A_{n} U
$$

where $U$ is the shear lag reduction factor and is calculated by:

$$
U=1-\frac{\bar{x}}{l}
$$

where $\bar{x}$ is the eccentricity of connection and $l$ is the length where force transfer occurs as shown in Figure 1a for an angle member with one row of three bolts. The net area is calculated as [19]:

$$
A_{n}=A_{g}-\sum\left(d_{n}+1 / 8\right) t
$$

where $d_{n}$ is the nominal hole diameter and $t$ is the thickness of the tension member.

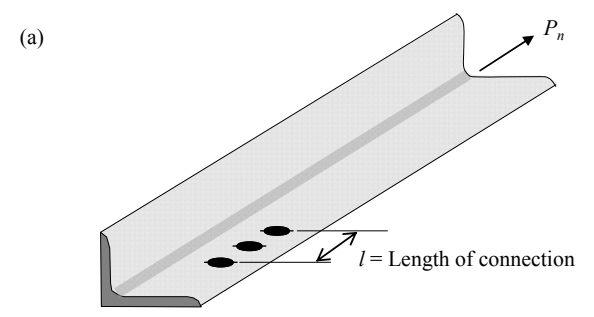

(b)

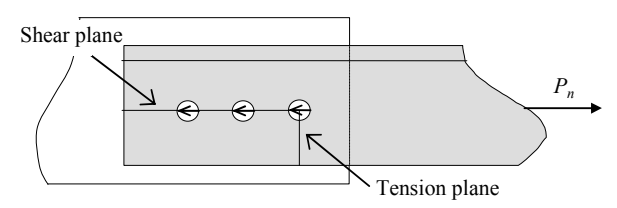

Fig. 1: (a) A standard AISC angle section, and (b) the shear and tension planes in block shear failure of the angle section.

In some cases, the strength of tension members is controlled by block shear strength, where the failure of a member may occur along a path involving tension on one plane and shear on a perpendicular plane [19] as shown in Figure 1b for an angle member with one row of bolts. The AISC specifies that the block strength, $P_{n b}$, is calculated as [7]:

$$
P_{n b}=0.6 F_{u} A_{n v}+U_{b s} F_{u} A_{n t} \leq 0.6 F_{y} A_{g v}+U_{b s} F_{u} A_{n t}
$$

where $A_{g v}$ is the gross area subject to shear, $A_{n v}$ is the net area subject to shear, $A_{n t}$ is the net area subject to tension, and $U_{b s}=$ 1 or 0.5 and is 1 for most tension members.

\section{NOMINAL STRENGTH OF TRUSS MEMBERS UNDER COMPRESSIVE LOADS}

The nominal compressive strength, $P_{n c}$, can be calculated as [7]:

$$
P_{n c}=F_{c r} A_{g}
$$

where $F_{c r}$ is the critical flexural buckling stress, which is calculated as per [7] as given in:

$$
F_{c r}= \begin{cases}{\left[0.658^{\frac{F_{y}}{F_{e}}}\right] F_{y}} & \text { for } \frac{K L}{r} \leq 4.71 \sqrt{\frac{E}{F_{y}}} \\ 0.877 F_{e} & \text { for } \frac{K L}{r}>4.71 \sqrt{\frac{E}{F_{y}}}\end{cases}
$$

where $E$ is the elastic modulus, $L$ is the length of the member, $K$ is the effective length factor which is 1.0 for truss members, $r$ is the minimum radius of gyration, and $F_{e}$ is the elastic (Euler) buckling stress calculated as [7]:

$$
F_{e}=\frac{\pi^{2} E}{\left(\frac{K L}{r}\right)^{2}}
$$




\section{STRUCTURAL RELIABILITY}

The safety margin for truss member $i, g_{i}$, also known as the performance function, is defined as the difference between the resistance of the truss member, $R_{i}$, and the internal force it is subjected to, $F_{i}$, and is given by:

$$
g_{i}=R_{i}-F_{i}
$$

The resistance of truss member $i$ can be expressed by:

$R_{i}= \begin{cases}\min \left[P_{n y}, P_{n r}, P_{n b}\right] & \text { for tension members } \\ P_{n c} & \text { for compression members }\end{cases}$

The reliability associated with failure of truss member $i$ due to the action of the internal load $F_{i}$ may be represented by the reliability index, $\beta_{f i}$, which is given by [20]:

$$
\beta_{f i}=\frac{\mu_{R i}-\mu_{F i}}{\sqrt{\sigma_{R i}^{2}+\sigma_{F i}^{2}}}
$$

where $\mu_{R i}, \mu_{F i}$ are the means of the resistance and internal force for truss member $i$, respectively, and $\sigma_{R i}, \sigma_{F i}$ are the standard deviations of the resistance and internal force for truss member $i$, respectively. Similarly, the reliability index, $\beta_{d j}$, associated with the exceeding of truss joint $j$ to an allowable displacement limit, $d_{\text {all }}$, is given by:

$$
\beta_{d j}=\frac{d_{a l l}-a b s\left(\mu_{d j}\right)}{a b s\left(\sigma_{d j}\right)}
$$

where $\mu_{d j}$ and $\sigma_{d j}$ are the mean and standard deviation of the displacement of truss joint $j$, respectively. Standard deviation, $\sigma$, is related to the coefficient of variation, $V$, as given by:

$$
\sigma=V \mu
$$

It is assumed in the examples of this paper that the truss members are each connected to the joint by one line of three bolts of $3 / 4$ inch diameter. The modulus of elasticity, $E_{i}$, the yield stress, $F_{y i}$, and the ultimate tensile stress, $F_{u i}$, for truss member $i$ are all treated as normally distributed random variables with coefficient of variation 0.05 and means, 29000 $\mathrm{ksi}, 50 \mathrm{ksi}$, and $65 \mathrm{ksi}$, respectively. The cross-sectional area, $A_{i}$, the eccentricity of connection, $\bar{x}$, and the radius of gyration, $r_{i}$, for truss member $i$ are also treated as normally distributed random variables with coefficient of variation 0.05 and means taken from the AISC tables for each angle section selected.

\section{IMPROVED FIREFLY ALGORITHM}

The FA is one of the newest algorithms which mimics the social behavior of fireflies based on their flashing characteristics. Each firefly movement is based on absorption of the other one's flash [21]. Since its appearance, it has been through several enhancements and it has attracted many applications [15]. The light intensity, $I(r)$, can be assumed to vary according to the inverse square law. For a medium with a fixed light absorption coefficient, the light intensity varies with the distance from the source, $d$, as [21]:

$$
I(r)=I_{0} e^{-\alpha d^{2}}
$$

where $I_{0}$ is the intensity at the source and $\alpha$ is the light absorption coefficient. In a similar manner, the attractiveness of a firefly, $\beta$, varies with the distance, $r_{i j}$, between firefly $i$ and firefly $j$ and can be calculated as [15]:

$$
\beta=\beta_{0} e^{-\gamma r^{2}}
$$

where $\beta_{0}$ is the attractiveness at distance $r=0, \gamma$ is the light absorption coefficient and $r$ is the Cartesian distance between firefly $i$ and $j$.

During the iterations of the FA, a pair-wise comparison of brightness of fireflies is conducted, where the one with lower light intensity moves toward the brighter one. The movement at iteration $t$ of firefly $i$, which is attracted by a brighter firefly $j$, is given by [15]:

$$
x_{i}^{(t+1)}=x_{i}^{(t)}+\beta\left(x_{j}^{(t)}-x_{i}^{(t)}\right)+\alpha_{0} \theta^{t} \varepsilon^{(t)}
$$

where $\alpha_{0}$ is the initial randomization parameter, $\theta$ is the randomization reduction constant, and $\varepsilon$ is a vector of random numbers drawn from a Gaussian distribution or uniform distribution in the range $[0,1]$.

In [3] an improvement to (17) was proposed. For design optimization problems with discrete design variables that can assume the index value of a standard section from a list of $p$ standard sections $x_{i}=\{1,2, \ldots, p\}$, the improved equation is formulated as [3]:

$$
x_{i}^{(t+1)}=\operatorname{INT}\left[x_{i}^{(t)}+\beta\left(x_{j}^{(t)}-x_{i}^{(t)}\right)+\alpha_{0} \theta^{t}(p-1)\left(\varepsilon^{(t)}-0.5\right)\right]
$$

\section{MODIFICATION OF THE IMPROVED FIREFLY ALGORITHM}

In probabilistic and reliability analysis of practical problems, determining the exact solution is most likely unachievable. Most known solution techniques suitable for practical problems are numerical and approximate at best. In some reliability analysis techniques, especially simulationbased techniques, one may repeat the solution of the same problem and obtain different results each time, albeit with a very small difference.

In problems with integer design variables, (18) may lead to the same value for $x^{t}$ and $x^{(t+1)}$ in some fireflies with high brightness during advanced iterations. If the brightness of a firefly is recalculated in a new generation and the new value is slightly different from that in the previous generation, this slight difference in brightness may cause the firefly to cross a constraint. Accordingly, the firefly will be penalized by assigning a large value to its objective function and thus its brightness will diminish compared to other fireflies. Hence, chances are that the value of the optimum solution in one iteration may be found larger than the value of the optimum solution of the previous iteration. It should be emphasized that this issue is only encountered in problems with probabilistic constraints. In problems with deterministic constraints, if the firefly is maintained in the following iteration, its brightness of course remains unchanged even if the objective functions and constraints are re-evaluated. 
It is proposed herein to introduce a modification to the IFA to solve problems with probabilistic constraints. It is proposed that for each iteration $t>1$ and within the step where the brightness is compared for the updated swarm of $n$ fireflies, the best firefly from the previous iteration $t-1$ is included in the comparison and the best $n$ fireflies are selected. The examples of this paper show that this modification improves the performance of the IFA and accelerates its convergence when used in probabilistic and RBDO problems.

\section{THE OPTIMIZATION PROBLEM FORMULATION}

In the examples of this paper, the members are selected from the AISC standard tables for angle sections. These are 127 angles and are arranged in this paper in a descending order of their weights (in pound per unit foot) in order to improve the optimization process. According to this order, each section is given an $i d$, such that the heaviest section takes $i d$ number 1 and the lightest takes $i d$ number 127 . The design variables in this paper are the section id numbers for the truss members. Each truss member, or group of members, is assigned an integer design variable, where its value determines the AISC angle section to be selected for it. In other words, truss member $i$ is assigned a design variable $x_{i}$ that is allowed to take one of the integer values from the set $\{1,2, \ldots ., 127\}$, which represent the $i d$ numbers of the AISC angle sections considered. In the firefly algorithm, the design variables are represented by swarms of fireflies. Hence, once the position of a firefly is determined, the corresponding discrete value for each section property is determined from the AISC tables.

The objective of the optimization problems in this paper is to minimize the weight of the structure. The weight of truss member $i$ is calculated as the weight per unit foot of its AISC section with $x_{i}$ id number, $W\left(x_{i}\right)$, multiplied by the length of this member, $L_{i}$, in feet. The constraints of the optimization problem include the reliability index associated with failure of the truss members due to the internal forces in each of these members, $\beta_{f i}$, and the reliability index associated with the displacement of each node in each direction, $\beta_{d i}$. A lower limit is assigned for $\beta_{f i}$, and $\beta_{d i}$ and they are given values of $\beta_{f l}$, and, $\beta_{d l}$, respectively. The optimization problem can now be formulated as follows:

$$
\begin{aligned}
& \text { Find: } x_{1}, x_{2}, \ldots, x_{n} \in\{1,2, \ldots, 127\} \\
& \text { To Minimize: } \\
& \qquad \sum_{i=1}^{n} W\left(x_{i}\right) L_{i}
\end{aligned}
$$

$$
\begin{aligned}
& \text { Subject to: } \\
& \beta_{f i} \geq \beta_{f l} \text { for } i=1,2, \ldots, n \\
& \beta_{d j} \geq \beta_{d l}
\end{aligned}
$$

where $n$ is the number of members, or group of members, in the truss and $m$ is the number of truss nodes. In the examples of this paper, it is considered that $\beta_{f l}=\beta_{d l}=3.0$.

The solution of this optimization problem is an integrated process as shown in the flowchart presented in Figure 2. In the framework shown in this flowchart, a loop of optimization iterations of the firefly algorithm takes place. In each iteration, the brightness of the fireflies is determined by calculating the objective function and constraints. Evaluating the constraints requires reliability analysis of the truss. In order to conduct the reliability analysis, the samples of Latin hypercube are generated and each sample set is used as a realization of the truss and the load the truss is subjected to. The internal forces for each truss member are determined using the direct stiffness method to analyze each truss realization. The resistance of each truss member and for each realization is determined using the section strength equations presented in this paper. The distribution parameters of the truss member resistances and the internal forces in each member are then determined from these results in order to calculate the reliability indices for the truss.

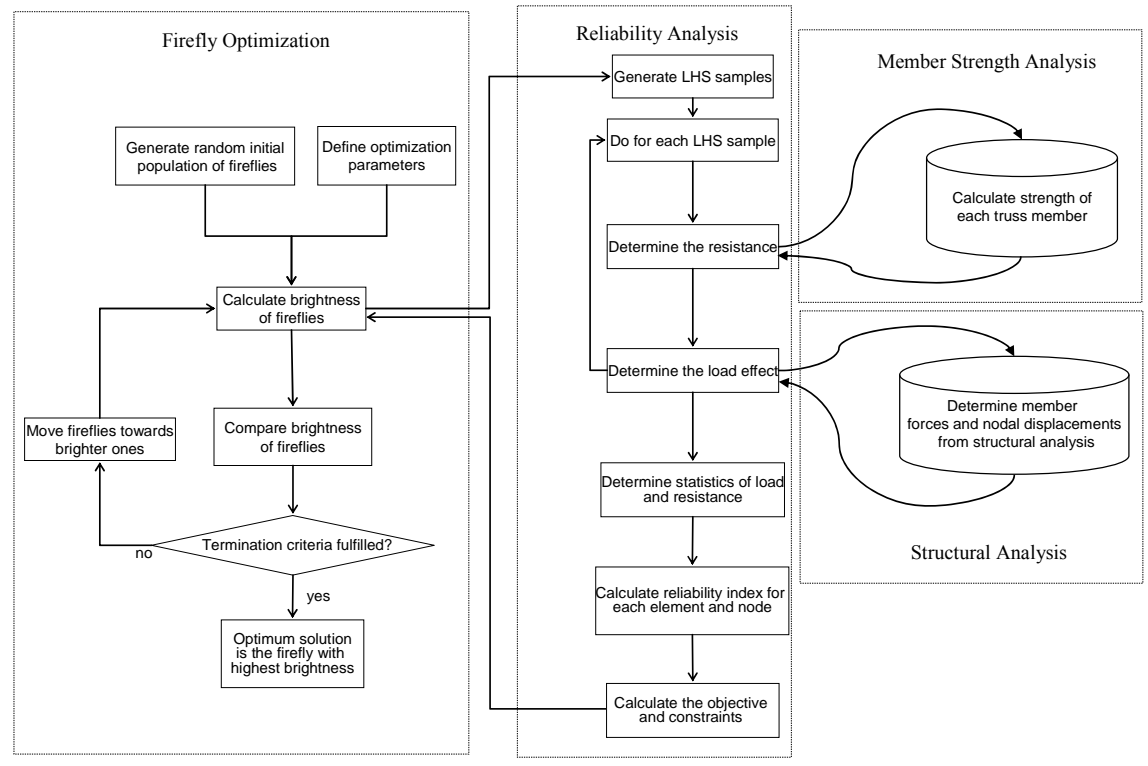

Fig. 2. Flowchart for the RBDO framework. 


\section{EXAMPLES}

\section{A. Ten-bar planar truss}

The design optimization of the truss shown in Figure 3 has been investigated in different forms in the literature $[3,4,6,9$, 22]. The load effects, $P_{1}$ and $P_{2}$, are each treated as a normally distributed random variable with mean and standard deviation 50 kip and 5 kips, respectively. An allowable displacement limit, $d_{\text {all }}$, is considered as 2 in. The results are shown in Figure 4. The details of the optimum solution are shown in Table I. The modified IFA was able to find the optimum solution at iteration 51 without problems. However, without the modification, the algorithm only reached the optimum solution at iteration 89. In addition, the best solution in iteration 4 was lost when the algorithm reached the end of iteration 5 and a best solution with higher objective value was registered at the end of iteration 5. The same occurred at iteration 28 .

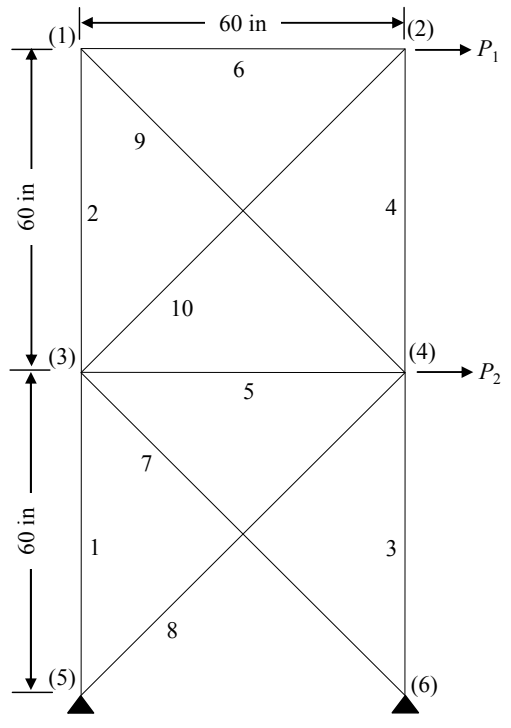

Fig. 3. Ten-bar planar truss.

TABLE I. OPTIMUM SOLUTION FOR THE TEN-BAR TRUSS.

\begin{tabular}{|c|c|}
\hline Truss Member & AISC Section Designation \\
\hline 1 & $\mathrm{~L} 6 \times 4 \times 5 / 16$ \\
\hline 2 & $\mathrm{~L} 3 \frac{1}{2} \times 3 \frac{1}{2} \times 5 / 16$ \\
\hline 3 & $\mathrm{~L} 3 \frac{1}{2} \times 3 \times 1 / 2$ \\
\hline 4 & $\mathrm{~L} 4 \times 3 \frac{1}{2} \times 5 / 16$ \\
\hline 5 & $\mathrm{~L} 4 \times 3 \frac{1}{2} \times 5 / 16$ \\
\hline 6 & $\mathrm{~L} 21 \frac{1}{2} \times 2 \times 3 / 8$ \\
\hline 7 & $\mathrm{~L} 3 \times 2 \times 1 / 2$ \\
\hline 8 & $\mathrm{~L} 4 \times 3 \times 3 / 8$ \\
\hline 9 & $\mathrm{~L} 2 \times 2 \times 1 / 8$ \\
\hline 10 & $\mathrm{~L} 3 \frac{1}{2} \times 3 \times 5 / 16$ \\
\hline
\end{tabular}

In iterations, the best solution was on the boundary of the constraint related to the reliability index associated with failure of truss member 1 . In order to illustrate the variability in the calculated reliability index for member 1 , the reliability calculations are conducted 1000 times in an analysis isolated from the optimization problem. Each time the reliability index is determined using the Latin hypercube sampling with 10000 samples. Accordingly, 1000 reliability indices were obtained. The histogram of the reliability index is shown in Figure 5. The figure shows, as expected, that there is a small variability in the reliability index. In fact, even though there is a chance that the reliability index has a value larger than 3.0, there is a higher probability that the reliability index takes values smaller than 3.0. This explains the loss of a best solution in an iteration; as one of the reliability indices that satisfied the constraints in that iteration may have a slightly different value when re-evaluated in the next iteration and thus violated a constraint.

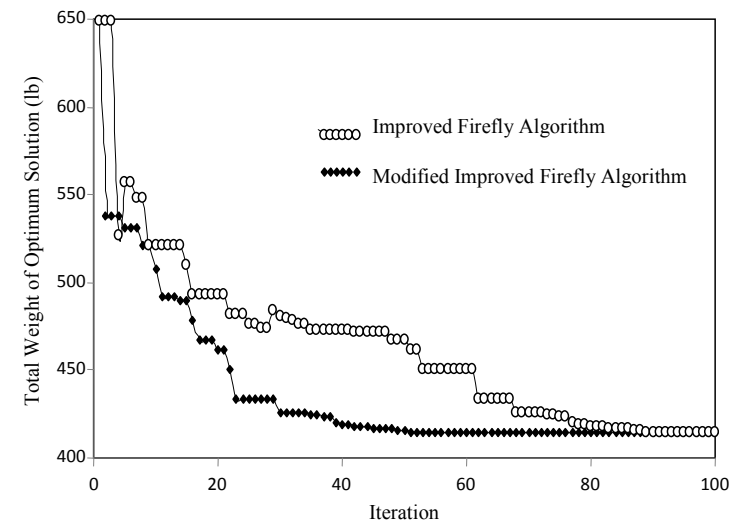

Fig. 4. Variation of the total weight of optimum solution with progress in iteration for the ten-bar truss.

The presence of variability in a reliability analysis is inevitable. The small variability in the resulting reliability index shown in Figure 5 is acceptable. Increasing the number of samples in the simulation may provide lower variability in the obtained reliability index. However, this will impose a computational burden on the optimization process. As shown in Figure 4, the suggested modification to the IFA was able to protect the algorithm from deviating from the converging solution and converge faster. The proven robustness of the IFA with the suggested modification is shown to be more efficient in handling RBDO problems with linked-discrete variables than the original IFA.

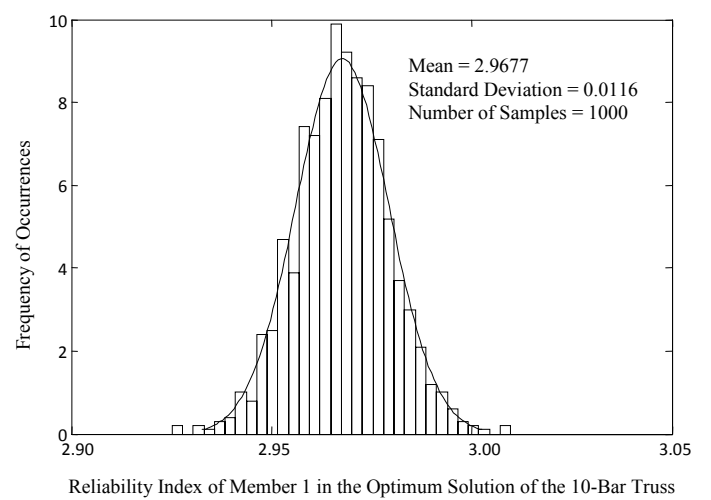

Fig. 5. Histogram of the reliability index associated with failure of member 1 in the ten-bar truss. 


\section{B. Twenty-five-bar space truss}

Figure 6 shows a twenty-five-bar space truss, which was used in different forms as a benchmark for investigating design optimization techniques in the literature [3,6,22]. The statistical parameters of the normally distributed load effects are shown in Table II. The 25 truss members are divided into 8 groups as follows: $\{1,2-5,6-9,10-11,12-13,14-17,18-21,22-25\}$.

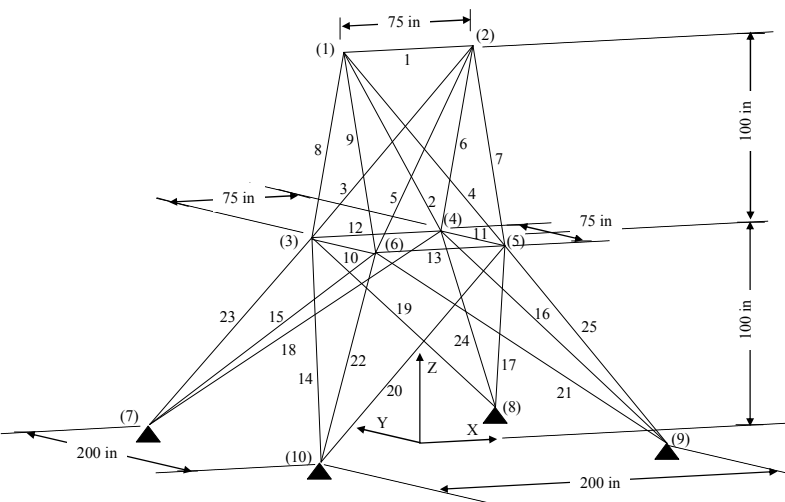

Fig. 6. Twenty-five-bar space truss.

TABLE II. LOAD EFFECT PARAMETERS FOR THE 25-BAR TRUSS.

\begin{tabular}{|c|c|c|c|}
\hline Node & Load Direction & Mean (kip) & Standard Deviation (kip) \\
\hline 1 & X & 2 & 0.2 \\
\hline 1 & Y & -20 & -2 \\
\hline 1 & Z & -20 & -2 \\
\hline 2 & Y & -20 & -2 \\
\hline 2 & Z & -20 & -2 \\
\hline 3 & X & 1 & 0.1 \\
\hline 6 & X & 1.2 & 0.12 \\
\hline
\end{tabular}

The optimization of the truss is investigated using the IFA with and without modification. An allowable displacement limit, $d_{\text {all }}$, is considered as $0.35 \mathrm{in}$. The results are shown in Figure 7. The details of the optimum solution are shown in Table III. It is clear that the modified IFA is able to find the optimum solution without problems. Without the modification, the algorithm reached the optimum solution later in the process than it did with the proposed modification. Furthermore, the best solution was lost in two different iterations without the proposed modification.

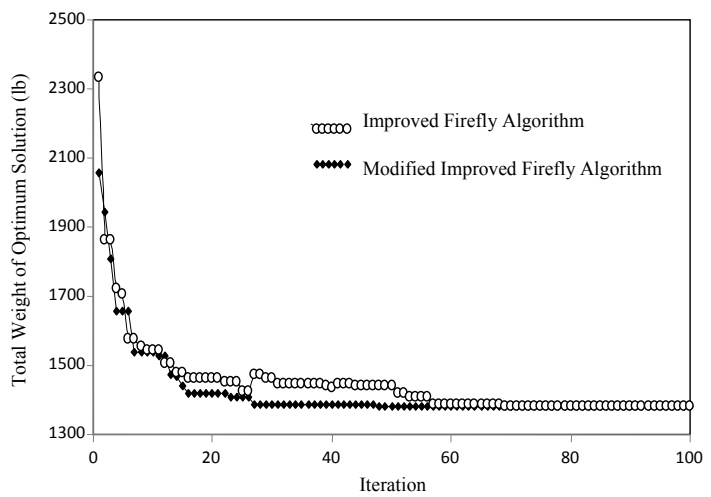

Fig. 7. Variation of the total weight of optimum solution with progress in iteration for the twenty-five-bar truss.

TABLE III. OPTIMUM SOLUTION FOR THE TWENTY-FIVE-BAR TRUSS.

\begin{tabular}{|c|c|}
\hline Truss Member Group & AISC Section Designation \\
\hline 1 & $\mathrm{~L} 4 \times 3 \frac{1}{2} \times 5 / 16$ \\
\hline 2 & $\mathrm{~L} 2 \frac{1}{2} \times 2 \times 3 / 16$ \\
\hline 3 & $\mathrm{~L} 3 \frac{1}{2} \times 2 \frac{1}{2} \times 1 / 2$ \\
\hline 4 & $\mathrm{~L} 3 \times 2 \times 1 / 4$ \\
\hline 5 & $\mathrm{~L} 31 \frac{1}{2} \times 3 \times 3 / 8$ \\
\hline 6 & $\mathrm{~L} 2 \frac{1}{2} \times 1 \frac{1}{2} \times 3 / 16$ \\
\hline 7 & $\mathrm{~L} 2 \frac{1}{2} \times 1 \frac{1}{2} \times 3 / 16$ \\
\hline 8 & $\mathrm{~L} 6 \times 3 \frac{1}{2} \times 5 / 16$ \\
\hline
\end{tabular}

\section{Sixty-five bar truss bridge}

The truss bridge is shown in Figure 8. One design variable is used for each pair of symmetric truss members with a total of 33 design variables. The bars are numbered from left to right starting from the bottom chord then the top chord then the diagonals. The load $P$ is treated as a normally distributed random variable with coefficient of variation 0.1 and mean of 30 kips. An allowable displacement limit, $d_{\text {all }}$, is considered as $0.5 \mathrm{ft}$. The details of the optimum solutions obtained by the modified IFA are shown in Table IV. Also shown in the table are the results obtained by the genetic algorithm MI-LXPM, which was conducted using the global optimization toolbox of MATLAB [24]. Also, using this toolbox, the problem was attempted to be solved by simulated annealing, and pattern search methods. However, they failed to find optimum solutions even near those shown in Table IV. Furthermore, the gradient-based optimization methods in the optimization toolbox of MATLAB [25] were tested for solving this problem.

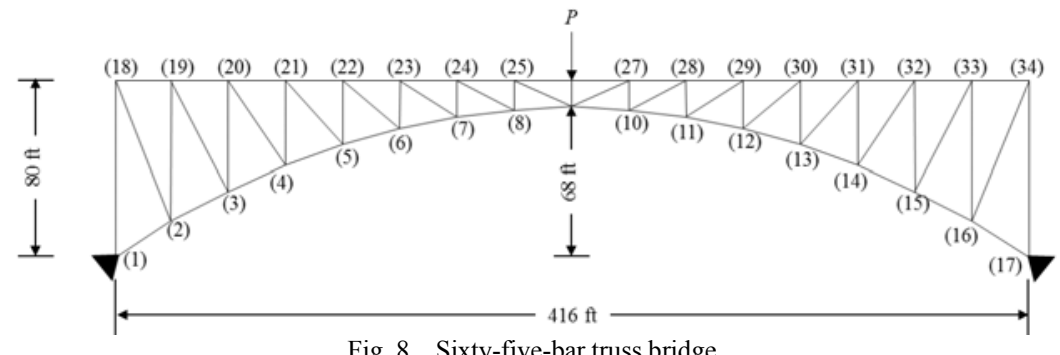

Fig. 8. Sixty-five-bar truss bridge. 
TABLE IV. OPTIMUM SOLUTION FOR THE TRUSS-BRIDGE.

\begin{tabular}{|c|c|c|}
\hline \multirow{2}{*}{ Truss Members } & \multicolumn{2}{|c|}{ AISC Section Designation } \\
\hline & Modified IFA & GA (MI-LXPM) \\
\hline $1-16$ & $\mathrm{~L} 6 \times 3 \frac{1}{2} \times 5 / 16$ & $\mathrm{~L} 4 \times 4 \times 3 / 4$ \\
\hline $2-15$ & $\mathrm{~L} 6 \times 4 \times 3 / 8$ & $\mathrm{~L} 4 \times 4 \times 1 / 2$ \\
\hline $3-14$ & $\mathrm{~L} 5 \times 5 \times 5 / 16$ & $\mathrm{~L} 4 \times 3 \times 1 / 2$ \\
\hline $4-13$ & $\mathrm{~L} 31 / 2 \times 31 / 2 \times 1 / 2$ & $\mathrm{~L} 31 / 2 \times 3 \times 7 / 16$ \\
\hline $5-12$ & $\mathrm{~L} 6 \times 3 \frac{1}{2} \times 1 / 2$ & $\mathrm{~L} 4 \times 3 \times 1 / 2$ \\
\hline $6-11$ & $\mathrm{~L} 6 \times 3 \frac{1}{2} \times 3 / 8$ & $\mathrm{~L} 8 \times 4 \times 5 / 8$ \\
\hline $7-10$ & $\mathrm{~L} 4 \times 3 \frac{1}{2} \times 5 / 16$ & $\mathrm{~L} 4 \times 3 \frac{1}{1} 2 \times 5 / 16$ \\
\hline $8-9$ & $\mathrm{~L} 4 \times 3 \times 5 / 16$ & $\mathrm{~L} 3 \frac{1}{2} \times 3 \times 1 / 2$ \\
\hline $17-32$ & $\mathrm{~L} 4 \times 3 \times 1 / 4$ & $\mathrm{~L} 4 \times 3 \times 5 / 16$ \\
\hline $18-31$ & $\mathrm{~L} 3 \times 3 \times 3 / 16$ & $\mathrm{~L} 3 \times 3 \times 3 / 8$ \\
\hline $19-30$ & $\mathrm{~L} 5 \times 3 \frac{1}{2} \times 5 / 16$ & $\mathrm{~L} 2 \times 2 \times 5 / 16$ \\
\hline $20-29$ & $\mathrm{~L} 3 \times 3 \times 5 / 16$ & $\mathrm{~L} 2 \times 2 \times 1 / 4$ \\
\hline $21-28$ & $\mathrm{~L} 5 \times 5 \times 5 / 16$ & $\mathrm{~L} 2 \times 2 \times 1 / 8$ \\
\hline $22-27$ & $\mathrm{~L} 2 \frac{1}{2} \times 2 \times 3 / 8$ & $\mathrm{~L} 2 \frac{1}{2} \times 2 \frac{1}{2} \times 3 / 8$ \\
\hline $23-26$ & $\mathrm{~L} 3 \frac{1}{2} \times 3 \frac{1}{2} \times 7 / 16$ & $\mathrm{~L} 3 \frac{1}{2} \times 3 \times 3 / 8$ \\
\hline $24-25$ & $\mathrm{~L} 8 \times 6 \times 1 / 2$ & $\mathrm{~L} 5 \times 5 \times 3 / 4$ \\
\hline $33-65$ & $\mathrm{~L} 2 \frac{1}{2} \times 1 \frac{1}{2} \times 3 / 16$ & $\mathrm{~L} 2 \times 2 \times 3 / 16$ \\
\hline $34-64$ & $\mathrm{~L} 2 \times 2 \times 3 / 16$ & $\mathrm{~L} 2 \frac{1}{2} \times 1 \frac{1}{2} \times 3 / 16$ \\
\hline $35-63$ & $\mathrm{~L} 2 \times 2 \times 1 / 8$ & $\mathrm{~L} 2 \times 2 \times 1 / 8$ \\
\hline $36-62$ & $\mathrm{~L} 31 / 2 \times 21 / 2 \times 1 / 4$ & $\mathrm{~L} 2 \times 2 \times 1 / 4$ \\
\hline $37-61$ & $\mathrm{~L} 2 \times 2 \times 1 / 8$ & $\mathrm{~L} 2 \frac{1}{2} \times 2 \times 1 / 4$ \\
\hline $38-60$ & $\mathrm{~L} 3 \times 3 \times 3 / 16$ & $\mathrm{~L} 2 \times 2 \times 3 / 16$ \\
\hline $39-59$ & $\mathrm{~L} 4 \times 4 \times 3 / 8$ & $\mathrm{~L} 21 / 2 \times 2 \times 3 / 16$ \\
\hline $40-58$ & $\mathrm{~L} 2 \times 2 \times 3 / 8$ & $\mathrm{~L} 3 \times 2^{1 / 2} \times 5 / 16$ \\
\hline $41-57$ & $\mathrm{~L} 3 \times 2 \times 1 / 2$ & $\mathrm{~L} 4 \times 4 \times 1 / 4$ \\
\hline $42-56$ & $\mathrm{~L} 2 \times 2 \times 1 / 8$ & $\mathrm{~L} 3 \times 2 \times 5 / 16$ \\
\hline $43-55$ & $\mathrm{~L} 4 \times 3 \times 1 / 2$ & L $3 \frac{1}{2} \times 2 \frac{1}{2} \times 1 / 4$ \\
\hline $44-54$ & L $31 / 2 \times 21 / 2 \times 3 / 8$ & $\mathrm{~L} 2 \times 2 \times 5 / 16$ \\
\hline $45-53$ & $\mathrm{~L} 4 \times 4 \times 1 / 4$ & $\mathrm{~L} 2^{1 / 2} \times 2^{1 / 1} / 2 \times 3 / 8$ \\
\hline $46-52$ & $\mathrm{~L} 4 \times 3 \times 3 / 8$ & $\mathrm{~L} 3 \times 2 \times 3 / 8$ \\
\hline $47-51$ & $\mathrm{~L} 4 \times 3 \times 5 / 16$ & $\mathrm{~L} 5 \times 3 \times 7 / 16$ \\
\hline $48-50$ & L $5 \times 3 \frac{1}{2} \times 5 / 16$ & $\mathrm{~L} 4 \times 3 \times 5 / 8$ \\
\hline 49 & L3 $1 \frac{1}{2} \times 3 \frac{1}{2} \times 3 / 8$ & $\mathrm{~L} 21 / 2 \times 2 \times 1 / 4$ \\
\hline Weight (lb) & 14624 & 14344 \\
\hline
\end{tabular}

As expected, these methods couldn't solve the problem either. Accordingly, the only optimization methods capable of handling this problem, among those attempted by the author, were the modified IFA and the MI-LXPM. Table IV shows that the total weight of the optimum solution obtained by MILXPM is slightly lower than that obtained by the modified IFA. Figure 9 shows the improvement in the total weight of the optimum solution with progress in iteration. It is clear from the figure that even though the MI-LXPM was capable of finding a slightly better solution than that obtained by the modified IFA, the latter method converged to its optimum solution at a fraction of the computational cost required by the former. Thus, it can be concluded from this example that the modified IFA was capable of finding a reasonable solution, in a reasonable amount of computations, relative to the MI-LXPM.

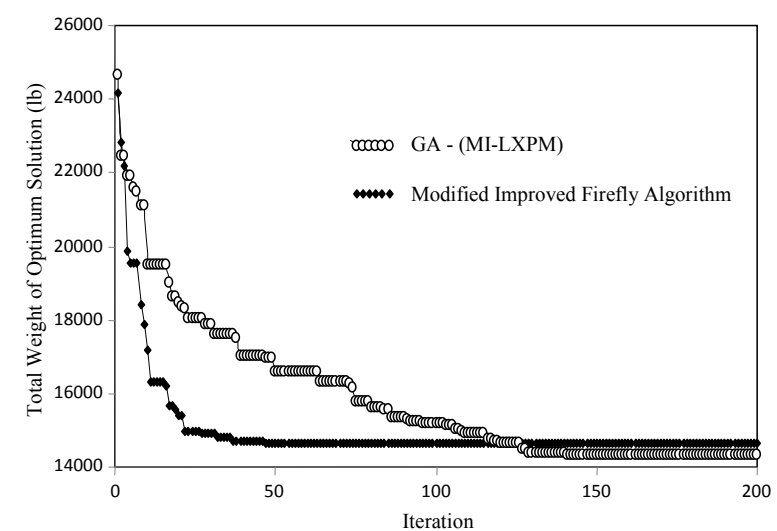

Fig. 9. Variation of the total weight of optimum solution with progress in iteration for the sixty-five-bar truss bridge.

\section{CONCLUSIONS}

In this paper, the structural reliability of trusses was considered in the design optimization process. The sections were selected from the AISC standard tables and thus the design variables that represent the properties of the section were linked-discrete. The IFA was used for the optimization. Latin hypercube sampling was used to determine the structural reliability. It was found that despite the well-known strength of the IFA in solving deterministic optimization problems, it is unstable when used in probabilistic and reliability-based optimization problems. A modification to the IFA was proposed in order to stabilize it for use in probabilistic and reliability-based design optimization problems. The proven robustness of the IFA with the proposed modification is shown to be efficient in handling the reliability-based optimum design problems with linked-discrete variables. Without the proposed modification, the IFA reached the optimum solution later than it did with the modification. Furthermore, it was shown by applying the presented approach to a sixty-five bar truss bridge and comparing the results with those from other methods that the modified IFA was capable of finding a reasonable solution, in a reasonable amount of computations, compared to the MILXPM. It should be emphasized that the main drawback of this approach is the computational cost of conducting the reliability analysis. Therefore, it is imperative that saving in computational cost during the optimization process should be a goal in a design optimization problem of this type. Even though the MI-LXPM found a better solution in the truss bridge example, this solution was found in about triple the computational cost of that required by the modified IFA to obtain its optimum solution. Perhaps this conclusion should encourage future attempts to create a hybrid algorithm, where the speed of convergence of the modified IFA is exploited at the beginning of the optimization process, then the MI-LXPM is used to improve the optimum solution.

\section{REFERENCES}

[1] P. Venkataraman, Applied Optimization with MATLAB Programming, John Wiley \& Sons, Inc. Hoboken, NJ, 2009 
[2] J. S. Arora, M. W. Huang, C. C. Hsieh, "Methods for Optimization of Nonlinear Problems with Discrete Variables: a Review", Structural Optimization, Vol. 8, No. 2/3, pp. 69-85, 1994

[3] A. Baghlani, M. H. Makiabadi, M. Sarcheshmehpour, "Discrete Optimum Design of Truss Structures by an Improved Firefly Algorithm”, Advances in Structural Engineering, Vol. 17, No. 10, pp. $1517-1530,2014$

[4] M. Alapati, "Discrete Optimization of Truss Structure Using Genetic Algorithm", International Journal of Recent Development in Engineering and Technology, Vol. 3, No. 1, pp. 105-111, 2014

[5] A. H. Gandomi, X. S. Yang, A. H. Alavi, "Mixed Variable Structural Optimization using Firefly Algorithm", Computers and Structures, Vol. 89, No. 23/24, pp. 2325-2336, 2011

[6] S. Mathakari, P. Gardoni, "Reliability-Based Optimal Design of Electrical Transmission Towers Using Multi-Objective Genetic Algorithms", Computer-Aided Civil and Infrastructure Engineering, Vol. 22, No. 4, pp. 282-292, 2007

[7] AISC Manual of Steel Construction, 14th Edition, American Institute of Steel Construction, Chicago, 2011

[8] J. S. Arora, M. W. Huang, "Discrete Structural Optimization with Commercially Available Sections: a Review", Journal of Structural and Earthquake Engineering, Vol. 13, No. 2, pp. 93-110, 1996

[9] M. W. Huang, J. S. Arora, "Optimal Design of Steel Structures using Standard Sections", Structural Optimization, Vol. 14, No. 1, pp. 24-37, 1997

[10] Y. Aoues, A. Chateauneuf, "Benchmark Study of Numerical Methods for Reliability-Based Design Optimization", Structural and Multidisciplinary Optimization, Vol. 41, No. 2, pp. 277-294, 2010

[11] A. Strauss, T. Zimmermann, D. Lehky, D. Novak, Z. Kersner, "Stochastic Fracture-Mechanical Parameters for the Performance-Based Besign of Concrete Structures", Structural Concrete, Vol. 15, No. 3, pp. 380-394, 2014

[12] N. M. Okasha, D. M. Frangopol, "Efficient Method Based on Optimization and Simulation for the Probabilistic Strength Computation of the Ship Hull”, Journal of Ship Research, Vol. 54, No. 4, pp. 1-13, 2010

[13] N. M. Okasha, D. M. Frangopol, A. Deco, "Integration of Structural Health Monitoring in Life-Cycle Performance Assessment of Ship Structures Under Uncertainty", Marine Structures, Vol. 3, No. 23, pp. 303-321, 2010

[14] K. Deb, Multi-Objective Optimization using Evolutionary Algorithms, Wiley, New York, 2001

[15] X. S. Yang, X. He, "Firefly Algorithm: Recent Advances and Applications", International Journal of Swarm Intelligence, Vol. 1, No. 1,pp. 36-50, 2013

[16] S. Kirkpatrick, C. D. Gelatt, M. P. Vecchi, "Optimization by Simulated Annealing', Science, Vol. 220, No. 4598, pp. 671-680, 1983

[17] P. Thanedar, G. Vanderplaats, "Survey of Discrete Variable Optimization for Structural Design", Journal of Structural Engineering, Vol. 121, No. 2, pp. 301-306, 1995

[18] K. Deep, K. P. Singh, M. L. Kansal, C. Mohan, "A real coded genetic algorithm for solving integer and mixed integer optimization problems", Applied Mathematics and Computation, Vol. 212, No. 2, pp. 505-518, 2009

[19] J. C. McCormac, S. F. Csernak, Structural Steel Design, 5th Edition. Prentice Hall, Upper Saddle River, NJ, 2012

[20] A. H. Ang, W. H. Tang, Probability Concepts in Engineering Planning and Design: Decision, Risk and Reliability, Volume II, John Wiley and Sons, NY, 1984

[21] J. Farkas, K. Jarmai, Optimum Design of Steel Structures, SpringerVerlag, Berlin Heidelberg, 2013

[22] S. Rajeev, C. Krishnamoorthy, "Discrete Optimization of Structures Using Genetic Algorithms", Journal of Structural Engineering, Vol. 118, No. 5, pp. 1233-1250, 1992

[23] R. Nakib, "Deterministic and Reliability-Based Optimization of Truss Bridges", Computers and Structures, Vol. 65, No. 5, pp. 767-775, 1997

[24] MathWorks, Global Optimization Toolbox - User's Guide, 2015

[25] MathWorks, Optimization ToolboxTM User's Guide, 2015 